\title{
MicroRNA-16 suppresses epithelial-mesenchymal transition-related gene expression in human glioma
}

\author{
QIN WANG ${ }^{1,2}, \mathrm{XU} \mathrm{LI}^{1,2}$, YU ZHU ${ }^{1,2}$ and PING YANG ${ }^{1,2}$ \\ ${ }^{1}$ Department of Clinical Laboratory, Tianjin Huanhu Hospital; \\ ${ }^{2}$ Tianjin Key Laboratory of Cerebral Vessels and Neural Degeneration, Tianjin 300060, P.R. China
}

Received October 14, 2013; Accepted June 10, 2014

DOI: $10.3892 / \mathrm{mmr} .2014 .2583$

\begin{abstract}
Glioma is one of the most prevalent types of brain tumor and is associated with the highest mortality rate of all CNS cancers. Epithelial-mesenchymal transition (EMT) has been recognized as an important factor in tumor metastasis. Previously, it has been demonstrated that microRNA-16 (miR-16) has an important role in tumor metastasis in human cancer cell lines. However, the role of miR-16 in epithelial-mesenchymal transition of human glioma cells remains unclear. In the present study, U87 and U251 glioma cell lines overexpressing miR-16 were established and it was identified that miR-16 suppressed invasion, adhesion, cell cycle, production of interleukin (IL)-6, IL-8 and transforming growth factor- $\beta$, and EMT-related gene expression, including vimentin, $\beta$-catenin and E-cadherin in miR-16 overexpressing U87 and U251 glioma cells. Furthermore, miR-16 suppressed EMT mainly through the downregulation of p-FAK and p-Akt expression, and nuclear factor- $\kappa \mathrm{B}$ and Slug transcriptional activity. Therefore, miR-16 may be an important therapeutic target and predictor for glioma therapy.
\end{abstract}

\section{Introduction}

Glioma is a type of central nervous system (CNS) cancer that is associated with a high mortality rate. Glioma has a particularly poor prognosis, the worst of all CNS cancer types, aside from brain stem glioma. It is considered that the various characteristics of glioma, including high invasion and wide range of metastasis, are associated with epithelial-mesenchymal transition (EMT) $(1,2)$.

EMT is a basic physiological process in multicellular organism morphology. It refers to the transdifferentiation phenomenon of epithelial cells to mesenchymal cells. In this process, epithelial cells gradually obtain the mesenchymal

Correspondence to: Mrs. Ping Yang, Department of Clinical Laboratory, Tianjin Huanhu Hospital, 122 Xiqiangtai Road, Tianjin 300060, P.R. China

E-mail: tjhhhospitallab@126.com

Key words: microRNA-16, glioma, epithelial-mesenchymal transition phenotype by losing polarity and acquiring mobility. EMT is mainly characterized by epithelial cell loss of polarity and the acquisition of mesenchymal characteristics. Specifically, this process includes: Cellular adhesion-related gene expression was reduced, causing epithelial cells to lose their intercellular adhesion capability. This induces structural changes in cellular keratin, which in turn transforms the morphology of the epithelial cells to a spindle fibroblast shape. The cells then acquire the characteristics of mesenchymal or fibroblast cells, including expression of vimentin, $\mathrm{N}$-cadherin and fibronectin. EMT is involved in the occurrence and development of epithelial origin tumors, particularly in the process of cancer invasion and metastasis. Therefore, investigating the role of EMT in the formation and metastasis of tumors, as well as its regulatory mechanisms, has attracted notable attention in recent years.

microRNA (miRNA) is a type of non-coding single-stranded small molecule RNA with 21-25 nucleotides. The main function of microRNA is to regulate the expression of genes through translation inhibition, mRNA lysis and deadenylation. Furthermore, it has been reported to have numerous effects on cell proliferation, survival, apoptosis and cell cycle. With the advancement of the studies on miR-16, numerous antitumor characteristics of this miRNA have been revealed, including as a genetic therapy tool for tumor treatment. The present study investigated the inhibitory effects of miR-16 on EMT-related gene expression of U87 and U251 glioma cells and provided evidence that has potential applications for the development of novel genetic tumor therapies using miR-16.

\section{Materials and methods}

Cell culture and transfection. The U87 and U251 human glioma cell line were obtained from the American Type Culture Collection (Manassas, VA, USA). The cells were cultured in Dulbecco's modified Eagle's medium (DMEM containing $10 \%$ fetal bovine serum) under the conditions of $5 \% \mathrm{CO}_{2}, 37^{\circ} \mathrm{C}$ and saturated humidity. The cells were digested by $0.25 \%$ trypsin-EDTA.

Pre-miR-16 and scrambled negative control RNA (pre-miR-NC) were purchased from GenePharma and transfected by Lipofectamine 2000 (Invitrogen Life Technologies, Carslbad, CA, USA) according to the manufacturer's 
instructions. The miR-16 quantification assays were conducted using an mirVana ${ }^{\mathrm{TM}}$ qRT-PCR miRNA Detection kit (Ambion, Austin, TX, USA) according to the manufacturer's instructions. The groups were as follows: (i) U87 or U215 cells without treatment (control group); (ii) U87 or U215 cells transfected with negative control RNA (pre-miR-NC group); (iii) U87 or U215 cells transfected with pre-miR-16 (pre-miR-16 group).

Transwell assay to detect the cell invasion potential of human glioma cell lines. A total of $3 \times 10^{5}$ cells were seeded into the upper compartment of the Transwell kit (Coster, South Elgin, IL, USA) with Matrigel (BD Biosciences, Franklin Lakes, NJ, USA), $600 \mu 10.1 \%$ bovine serum albumin medium was added to the lower compartment and cells were cultured with $5 \% \mathrm{CO}_{2}$ at $37^{\circ} \mathrm{C}$ for $24 \mathrm{~h}$. Then, the cells were washed three times with cold phosphate-buffered saline, fixed, stained with hematoxylin and eosin and counted in at least five areas using a CX31 microscope (Olympus Corporation, Shimadzu, Japan) at a magnification of $\mathrm{x} 100$.

Cell adhesion assay to detect the cell adhesion potential of human glioma cell lines. A total of $5 \times 10^{5}$ cells were seeded into 96-well plates and incubated with $5 \% \mathrm{CO}_{2}$ in $37^{\circ} \mathrm{C}$ for $2 \mathrm{~h}$. The medium was discarded and the wells were washed four times in PBS. MTS dye (Promega Corporation, Madison, WI, USA) was added to the wells and the adhering cell rate was measured at $490 \mathrm{~nm}$ by a Bio-Rad microplate-reader $4 \mathrm{~h}$ later (Bio-Rad Laboratories, Hercules, CA, USA).

Flow cytometric assay to detect the cell cycle of human glioma cell lines. A total of $3 \times 10^{5}$ cells were seeded into 6-well plates and incubated with $5 \% \mathrm{CO}_{2}$ at $37^{\circ} \mathrm{C}$ for $24 \mathrm{~h}$. The cells were then collected and fixed with $75 \%$ cold alcohol at $4^{\circ} \mathrm{C}$ overnight. Then, the cells were centrifuged at $1,000 \mathrm{x} g$ for $5 \mathrm{~min}$ and incubated with RNase A and PI for $30 \mathrm{~min}$. The cell cycle stage of the tumor cells was measured by flow cytometry (FACSAria; BD Biosciences) at $488 \mathrm{~nm}$.

Enzyme-linked immunosorbent assay (ELISA) assay to detect the interleukin (IL)-6,IL-8 and transforming growth factor (TGF)- $\beta$ production in human glioma cell lines. A total of $3 \times 10^{5}$ cells were seeded into 6 -well plates and incubated with $5 \% \mathrm{CO}_{2}$ at $37^{\circ} \mathrm{C}$ for $24 \mathrm{~h}$. The medium was replaced with serum free DMEM and was incubated for an additional $24 \mathrm{~h}$. Then, the medium was collected and centrifuged at $1,000 \mathrm{x} \mathrm{g}$ for 5 min. The IL- 6, IL- 8 and TGF- $\beta$ production in tumor cells was measured by a human ELISA kit (R\&D Systems, Inc., Minneapolis, MN, USA) at $490 \mathrm{~nm}$.

Western blot analysis. The cells were lysed and centrifuged at $4^{\circ} \mathrm{C}$ and $14,000 \times \mathrm{g}$ for $5 \mathrm{~min}$, and the concentration of the extracted protein was measured using a Coomassie (Bradford) protein assay kit (Pierce Biotechnology, Inc., Rockford, IL, USA). The protein was separated by $12 \%$ SDS-PAGE and transferred onto a nitrocellulose membrane. The monoclonal antibodies against E-cadherin, vimentin, $\beta$-catenin, p-FAK and p-Akt were diluted at a ratio of 1:500 (Santa Cruz Biotechnology, Inc., Santa Cruz, CA, USA), and the $\beta$-actin monoclonal antibody was diluted at a ratio of 1:2,000 (Sigma, St. Louis, MO, USA) as an internal control at $4^{\circ} \mathrm{C}$

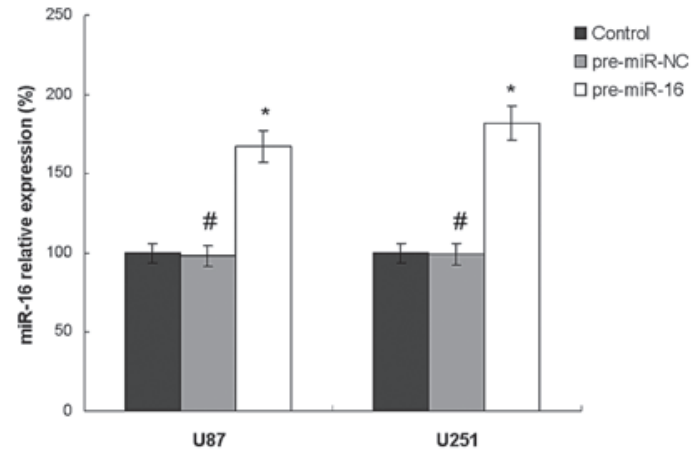

Figure 1. Expression of miR-16 in human glioma cell lines. The quantitative polymerase chain reaction results demonstrated that miR-16 was overexpressed in the U87 pre-miR-16 and U251 pre-miR-16 groups. The negative control RNA transfection group was the pre-miR-NC group and the untransfected cell line served as the control group. The values are presented as the mean \pm standard deviation, $\mathrm{n}=5$ pergroup. ${ }^{\#} \mathrm{P}<0.05$ and ${ }^{*} \mathrm{P}<0.05$, compared with the control group. miR-16, microRNA-16.

overnight. The membrane was washed using Tris-buffered saline with $0.1 \%$ Tween-20 and was added to $1: 1,000$ diluted horseradish peroxidase (HRP)-conjugated goat anti-rabbit secondary antibody (Santa Cruz Biotechnology, Inc.) at room temperature for $1 \mathrm{~h}$. Following washing, the membrane was visualized with the Phototope-HRP Western Blot Detection system (Cell Signaling Technology, Inc., Danvers, MA, USA).

Quantitative (q)PCR assay. The cells were collected and the total RNA was extracted by TRIzol (Invitrogen Life Technologies). GAPDH was used as the internal control. The total RNA was amplified using the following primers: Sense: 5'-ACAGCCCCGCCTTATGATT-3' and antisense: 5'-TCGGAACCGCTTCCTTCA-3' for E-cadherin; sense: 5'-TGGCACGTCTTGACCTTGAA-3' and antisense: 5'-GGT CATCGTGATGCTGAGAA-3' for vimentin; sense: 5'-TTT GAAGGCAGTCTGTCGTA-3' and antisense: 5'-TTATGC TTTGCACTACGTCCCTCCA-3' for $\beta$-catenin; and sense: 5'-ATGGAAATCCCATCACCATCTT-3' and antisense: 5'-CGCCCCACTTGATTTGG-3' for GAPDH. The amplification conditions were: Pre-denaturation, $94^{\circ} \mathrm{C}$ for $5 \mathrm{~min}$, a total of 35 cycles of amplification were conducted and each cycle consisted of $5 \mathrm{sec}$ at $95^{\circ} \mathrm{C}$ and $35 \mathrm{sec}$ at $65^{\circ} \mathrm{C}$.

Report gene assay. pNF-кB-luc, pSlug-luc and the CMV promoter Renilla luciferase internal standard (Beyotime, Shanghai, China) were transfected by Lipofectamine 2000 (Invitrogen Life Technologies, Carlsbad, CA, USA) to detect the transcriptional activity of nuclear factor (NF) $-\kappa B$ and Slug according to manufacturer's instructions. The ratio between the number of firefly luciferase excitation photon and the number of Renilla luciferase excitation photons was calculated.

Statistical analysis. The experimental data is expressed as the mean \pm standard deviation and was analyzed using SPSS 13.0 software (SPSS, Inc., Chicago, IL, USA). One-way analysis of variance was applied to compare the data of different groups and $\mathrm{P}<0.05$ was considered to indicate a statistically significant difference. Each experiment was repeated three times to obtain similar results. 

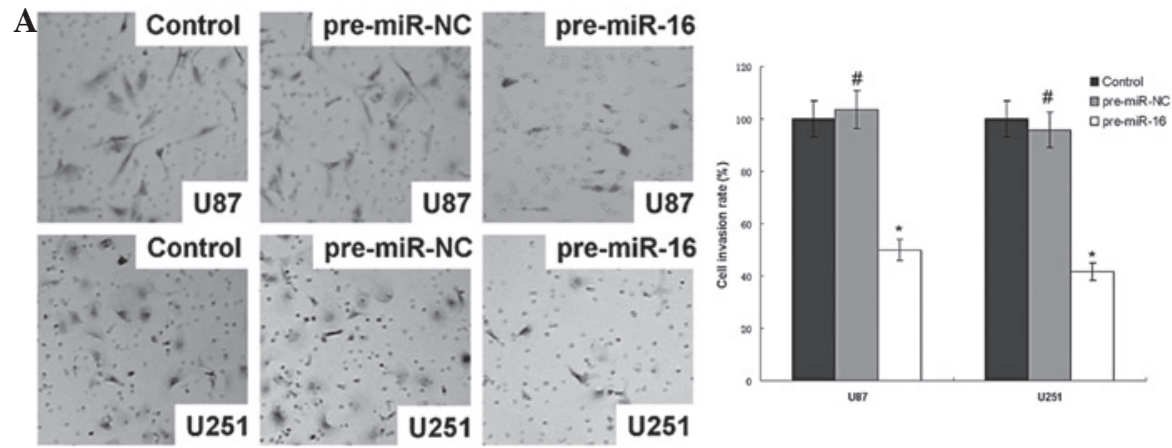

B
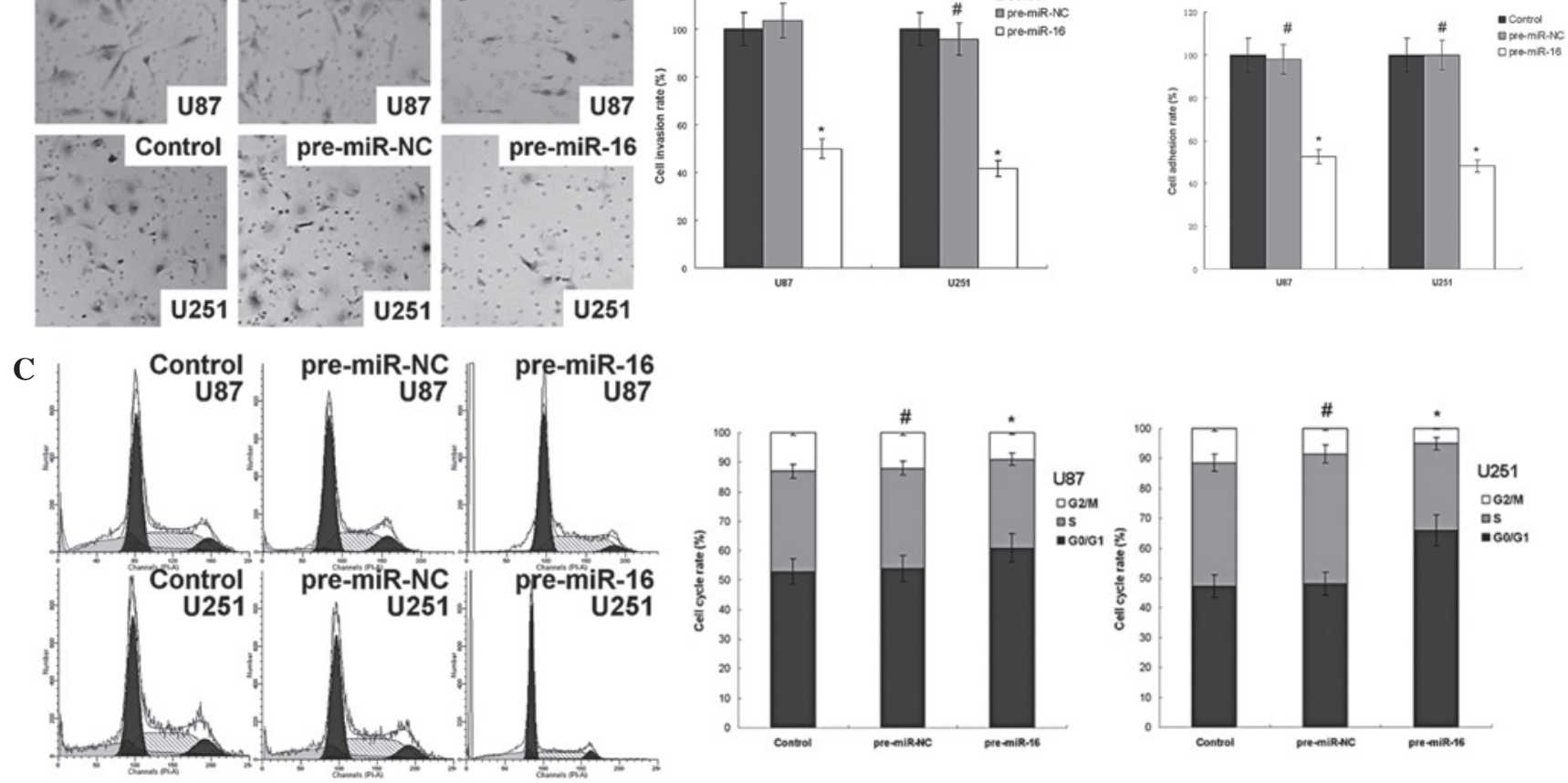

Figure 2. miR-16 regulates invasion and adhesion potential of human glioma cell lines in vitro. (A) The transwell assay results demonstrated that the invasion rate of the U87 pre-miR-16 and U251 pre-miR-16 groups were 50.0 and $41.6 \%$ compared with the control group, respectively. The values are presented as the mean $\pm \mathrm{SD}, \mathrm{n}=3$. (B) The adhesion rates of the U87 pre-miR-16 and U251 pre-miR-16 groups were 52.6 and $48.2 \%$ compared with control group, respectively. The values are presented as the mean $\pm \mathrm{SD}, \mathrm{n}=5$. (C) The G0/G1 phase of U87 pre-miR-16 and U251 pre-miR-16 groups were 61.5 and $66.3 \%$ and increased significantly compared with the control group at 53.2 and $47.3 \%$, respectively. The values are presented as the mean $\pm \mathrm{SD}, \mathrm{n}=3 .{ }^{*} \mathrm{P}<0.05$ and ${ }^{*} \mathrm{P}<0.05$, compared with the control group. miR-16, microRNA-16; ELISA, enzyme-linked immunosorbent assay; pre-miR-NC, scrambled negative control RNA; SD, standard deviation.
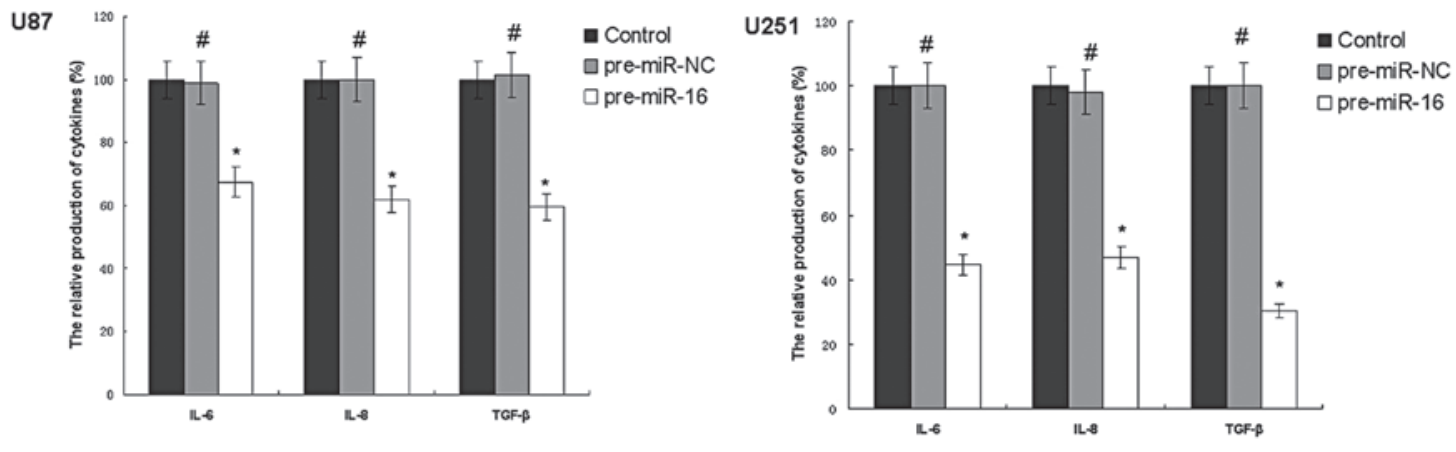

Figure 3. miR-16 regulates IL-6, IL-8 and TGF- $\beta$ production in human glioma cell lines in vitro. The ELISA results demonstrated that overexpressing miR-16 reduced the IL-6, IL-8 and TGF- $\beta$ production of U87 cells. The IL-6 production of the U87 pre-miR-16 and U251 pre-miR-16 groups was 67.5 and $44.6 \%$, the IL-8 production of the U87 pre-miR-16 and U251 pre-miR-16 groups was 62.3 and $46.8 \%$, the TGF- $\beta$ production of the U87 pre-miR-16 and U251 pre-miR-16 groups was 59.5 and $30.3 \%$ compared with control group, respectively. The values are presented as the mean \pm standard deviation, $n=5$. " $\mathrm{P}<0.05$ and "P<0.05, compared with the control group. miR-16, microRNA-16; IL, interleukin; TGF, transforming growth factor; pre-miR-NC, scrambled negative control RNA; ELISA, enzyme-linked immunosorbent assay.

\section{Result}

Expression of miR-16 in human glioma cell lines. The miR-16 expression levels in these cell lines were measured by qPCR (Fig. 1). The results showed that there was an increase in the expression levels of miR-16 in the pre-miR-16 group compared with those in the control group in U87 and U251 cell lines. The expression of miR-16 in U87 pre-miR-16 and U251 pre-miR-16 groups were 173.2 and $187.6 \%$ compared with that of the control group, respectively.
miR-16 regulates the invasion and adhesion potential of human glioma cell lines in vitro. As demonstrated in Fig. 2, miR-16 overexpression reduced the invasion and adhesion potential of U87 and U251 cells. The invasion rate of the U87 pre-miR-16 and U251 pre-miR-16 groups were 50.0 and $41.6 \%$ compared with the control group, respectively. The adhesion rate of the U87 pre-miR-16 and U251 pre-miR-16 groups were 52.6 and $48.2 \%$ compared with the control group, respectively. miR-16 overexpression also arrested the cell cycle in the G0/G1 phase, as revealed by the significant increase in the 

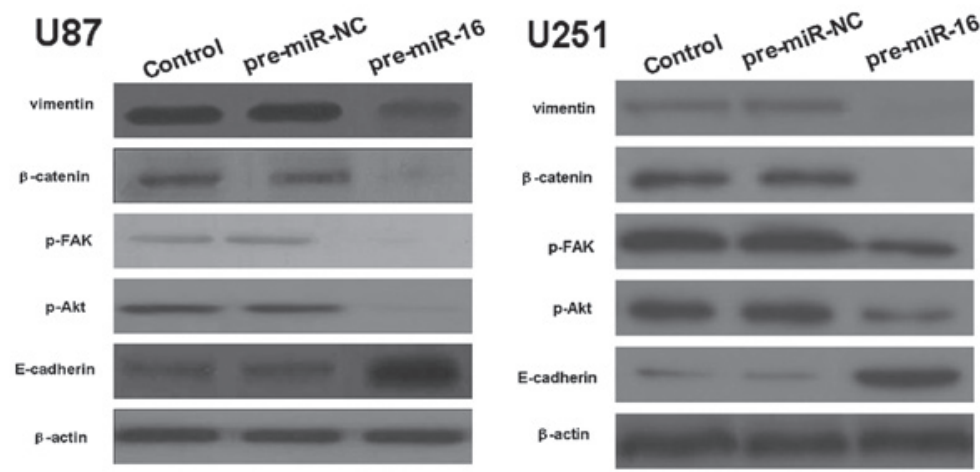

Figure 4. miR-16 regulates EMT-related protein expression in human glioma cell lines in vitro. The western blotting results demonstrated that the protein expression of vimentin, $\beta$-catenin, p-Akt and p-FAK in the U87 and U251 overexpressing cells was decreased significantly, compared with the negative control and control groups, while the expression of E-cadherin was increased. $\beta$-actin was used for normalization. miR-16, microRNA-16; EMT, epithelial-mesenchymal transition; pre-miR-NC, scrambled negative control RNA.
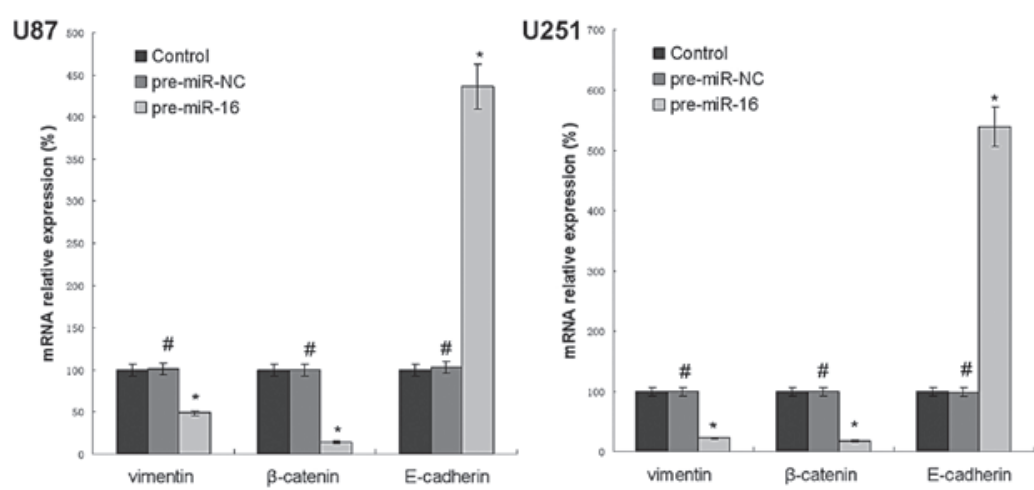

Figure 5. miR-16 regulates EMT-associated gene mRNA expression in human glioma cell lines in vitro. The quantitative polymerase chain reaction results demonstrated that the mRNA expression of vimentin and $\beta$-catenin in the U87 and U251 miR-16 overexpressing cells was decreased significantly, compared with the negative control and control groups, while the expression of E-cadherin was increased. GAPDH was used for normalization. The values are presented as the mean \pm standard deviation, $n=5 .{ }^{~} \mathrm{P}<0.05$ and ${ }^{*} \mathrm{P}<0.05$, compared with the control group. miR-16, microRNA-16; EMT, epithelial-mesenchymal transition; pre-miR-NC, scrambled negative control RNA.
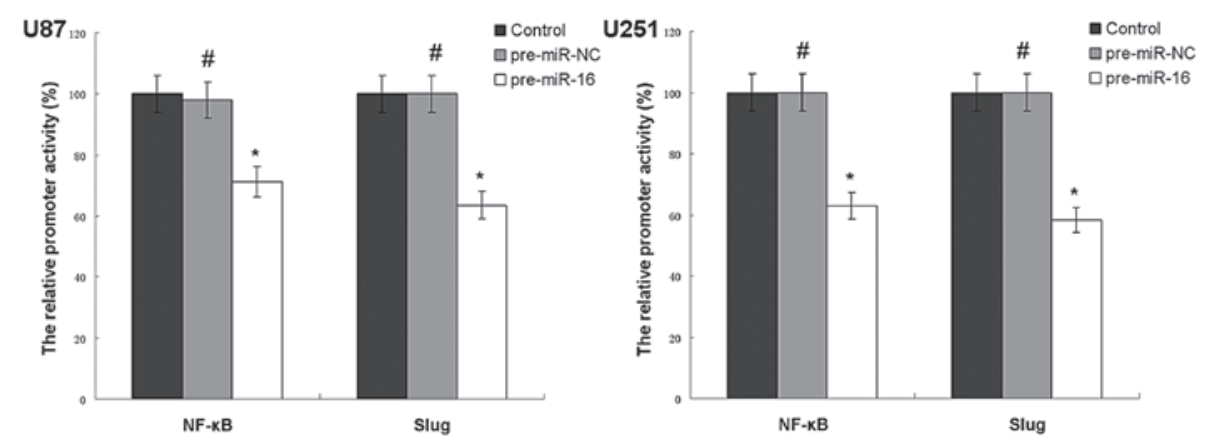

Figure 6. miR-16 regulates transcriptional activity of NF- $\mathrm{KB}$ of human glioma cell lines in vitro. The reporter gene assay demonstrated that the transcriptional activity of NF-kB and Slug in U87 and U251 miR-16 overexpressing cells were decreased significantly, compared with the negative control and control groups. The values are presented as the mean \pm standard deviation, $n=5$. ${ }^{\prime \prime} \mathrm{P}<0.05$ and ${ }^{*} \mathrm{P}<0.05$, compared with the control group. miR-16, microRNA-16; NF- $\mathrm{kB}$, nuclear factor- $\kappa \mathrm{B}$, pre-miR-NC, scrambled negative control RNA.

percentage of cells in the G0/G1 phase in the U87 pre-miR-16 and U251 pre-miR-16 groups, at 61.5 and $66.3 \%$ compared with the control group values of 53.2 and $47.3 \%$, respectively.

miR-16 regulates $I L-6, I L-8$ and TGF- $\beta$ production of human glioma cell lines in vitro. As demonstrated in Fig. 3, miR-16 overexpression reduced the IL- 6 , IL- 8 and TGF- $\beta$ production of $\mathrm{U} 87$ and $\mathrm{U} 251$ cells. The IL-6 production of the U87 pre-miR-16 and U251 pre-miR-16 groups was 67.5 and $44.6 \%$ compared with the control group, respectively. The IL-8 production of the U87 pre-miR-16 and U251 pre-miR-16 groups were 62.3 and $46.8 \%$, and the TGF- $\beta$ production of the U87 pre-miR-16 and U251 pre-miR-16 groups were 59.5 and $30.3 \%$ compared with the control group, respectively. 
miR-16 regulates EMT-related gene expression of human glioma cell lines in vitro. The EMT-related gene expression of human glioma cell lines in vitro was measured by western blotting and qPCR. The results revealed that the U87 and U251 miR-16 overexpressing groups had a significantly lower protein expression levels of vimentin, $\beta$-catenin, $p$-Akt and p-FAK, compared with control groups. By contrast, the protein expression levels of E-cadherin in the U87 and U251 miR-16 overexpressing groups were higher than the control groups (Fig. 4). In addition, the mRNA expression levels of vimentin, $\beta$-catenin and E-cadherin reflected the same trend in the U87 and $\mathrm{U} 251$ cell lines (Fig. 5).

miR-16 regulates $N F-\kappa B$ transcriptional activity of human glioma cell lines in vitro. As demonstrated in Fig. 6, miR-16 overexpression reduces the promoter activity of NF- $\mathrm{kB}$ and Slug in U87 and U251 cells. The promoter activity of NF- $\mathrm{kB}$ in the U87 pre-miR-16 and U251 pre-miR-16 groups were 71.3 and $62.9 \%$ compared with the control group, respectively. The promoter activity of Slug in the U87 pre-miR-16 and U251 pre-miR-16 groups was 63.5 and $58.2 \%$ compared with the control group, respectively.

\section{Discussion}

Previously, the correlation between EMT, and tumor invasion and metastasis has attracted notable attention. EMT refers to the process whereby epithelial cells interact with surrounding mesenchymal cells, decreasing the expression of cellular adhesion molecules, including E-cadherin, and leading to cell dispersion. Subsequently, the main component of the cytoskeleton gradually changes from keratin to vimentin resulting in its rearrangement, a decrease in cellular adhesion and the enhancement of cell mobility. Therefore, EMT enables non-invasive tumor cells to obtain invasion ability, which then accelerates the formation of local tumor invasion and distant metastasis (3).

E-cadherin and vimentin are important markers of EMT and are key for maintaining the epithelial phenotype and stabilizing inter-cellular contact (4-5). The present study has demonstrated that subsequent to the overexpression of miR-16, the mRNA and protein expression of E-cadherin in tumor cells was significantly increased, while the mRNA and the protein expression of vimentin was reduced in U87 and U251 cells. These data indicate that the inhibitory effect of miR-16 on the EMT process may be associated with the regulation of the expression of E-cadherin and vimentin in tumor cells. $\beta$-catenin, as a type of cellular adhesion and signal transduction molecule, has an important role in the maintenance of cellular structure and function. It may participate in cell adhesion as a cytoskeletal component and participate in gene transcription inside the nucleus. As an important member of the classic E-cadherin-catenin complex, $\beta$-catenin bonds with E-cadherin to participate in cell adhesion and connection.

Akt, FAK, NF- $\mathrm{kB}$, Slug, IL-6, IL-8 and TGF- $\beta$ also have a key role in the signal transduction pathway and upon activation may regulate downstream gene expression, which is associated with EMT (6-9). The effect of EMT on p-Akt and p-FAK protein expression, NF- $\mathrm{BB}$ and Slug transcriptional activity, and IL-6, IL-8 and TGF- $\beta$ production in tumor cells was investigated and it was identified that miR-16 affected the EMT process by preventing the excessive phosphorylation of FAK and Akt proteins, the transcriptional activity of NF- $\mathrm{KB}$ and Slug, and IL-6, IL-8 and TGF- $\beta$ production in tumor cells in the EMT process.

In conclusion, miR-16 is closely associated with EMT-related gene expression in human glioma and the present study may have established a theoretical basis for a future clinical treatment for human glioma.

\section{References}

1. Guo L, Qiu Y, Ge J and Zhou D: Glioblastoma multiforme with subcutaneous metastases, case report and literature review. J Korean Neurosurg Soc 52: 484-487, 2012.

2. Velpula KK, Dasari VR, Tsung AJ, Dinh DH and Rao JS: Cord blood stem cells revert glioma stem cell EMT by down regulating transcriptional activation of Sox 2 and Twist1. Oncotarget 2: 1028-1042, 2011

3. Techasen A, Loilome W, Namwat N, Dokduang H, Jongthawin J and Yongvanit P: Cytokines released from activated human macrophages induce epithelial mesenchymal transition markers of cholangiocarcinoma cells. Asian Pac J Cancer Prev 13 (Suppl): 115-118, 2012.

4. Gheldof A and Berx G: Cadherins and epithelial-to-mesenchymal transition. Prog Mol Biol Transl Sci 116: 317-336, 2013.

5. Liu T, Zhang X, Shang M, Zhang Y, Xia B, Niu M, Liu Y and Pang D: Dysregulated expression of Slug, vimentin, and E-cadherin correlates with poor clinical outcome in patients with basal-like breast cancer. J Surg Oncol 107: 188-194, 2013.

6. Chen CH, Chuang SM, Yang MF, Liao JW, Yu SL and Chen JJ: A novel function of YWHAZ/ $\beta$-catenin axis in promoting epithelial-mesenchymal transition and lung cancer metastasis. Mol Cancer Res 10: 1319-1331, 2012.

7. Ho JN, Jun W, Choue R and Lee J: I3C and ICZ inhibit migration by suppressing the EMT process and FAK expression in breast cancer cells. Mol Med Rep 7: 384-388, 2013.

8. Zhong D, Ran JH, Tang WY, Zhang XD, Tan Y, Chen GJ, Li XS and Yan Y: Mda-9/syntenin promotes human brain glioma migration through focal adhesion kinase (FAK)-JNK and FAK-AKT signaling. Asian Pac J Cancer Prev 13: 2897-2901, 2012.

9. Takenouchi T, Iwamaru Y, Imamura M, Fukuhara S, Sugama S, Sato M, Mochizuki N, Hashimoto M, Yokoyama T, Mohri S and Kitani H: Cytochalasin D enhances the accumulation of a protease-resistant form of prion protein in $\mathrm{ScN} 2$ a cells: involvement of PI3 kinase/Akt signalling pathway. Cell Biol Int 36: 1223-1231, 2012. 